\title{
A direct method and convergence analysis for some system of singular integro-differential equations
}

\author{
Iurie Caraus* Zhilin $\mathrm{Li}^{\dagger}$
}

\begin{abstract}
A class of singular integro-differential equations in Lebesgue spaces are studied. There are many applications of the singular integro-differential equations discussed in this paper. An example in modeling the stress distribution of an elastic medium with holes is discussed in the paper. Direct numerical schemes using a collocation method and a mechanical quadrature rule designed for the singular integro-differential equations are proposed for arbitrary smooth closed contours. Convergence analysis of these methods are given. Numerical examples are also provided.
\end{abstract}

Keywords: singular integro-differential equations, elasticity, collocation methods, mechanical quadrature rule.

\section{Introduction}

In this paper, we study the following system of singular integro-differential equations (SIDE)

$$
\begin{gathered}
(M x \equiv) \sum_{r=0}^{\nu}\left[\tilde{A}_{r}(t) x^{(r)}(t)+\tilde{B}_{r}(t) \frac{1}{\pi i} \int_{\Gamma} \frac{x^{(r)}(\tau)}{\tau-t} d \tau\right. \\
\left.+\frac{1}{2 \pi i} \int_{\Gamma} K_{r}(t, \tau) \cdot x^{(r)}(\tau) d \tau\right]=f(t), \quad t \in \Gamma,
\end{gathered}
$$

where $\tilde{A}_{r}(t), \tilde{B}_{r}(t)$, and $K_{r}(t, \tau),(r=\overline{0, \nu})$ are given $m$ by $m$ matrix functions, $f(t)$ is a given $m$ dimensional vector function, $x^{(0)}(t)=x(t)$ is a $m$ dimensional unknown vector function with up to $\nu$ 's continuous derivatives $\frac{d^{r} x(t)}{d t^{r}}(r=\overline{1, \nu})$, and $\Gamma$ is an arbitrary

${ }^{*}$ Department of Mathematics and Informatics, Moldova State University, Mateevici 60 str., Chisinau, Moldova, MD-2009, e-mail: caraush@mail.md

${ }^{\dagger}$ Center for Research in Scientific Computation \& Department of Mathematics, North Carolina State University, Raleigh, NC 27695, e-mail: zhilin@math.ncsu.edu 
smooth closed contour. We wish to find the solution $x(t)$ to equation (1) satisfying the condition

$$
\frac{1}{2 \pi i} \int_{\Gamma} x(\tau) \tau^{-k-1} d \tau=0, \quad k=\overline{0, \nu-1} .
$$

We will develop two direct methods to solve the system of singular integral equations (SIDE) (1)-(2) and provide the convergence analysis.

Singular integral equations (SIE) and SIDE have been used to model many physical problems, for examples, elasticity theory $[2,9,11,15,16,17,20]$, aerodynamics $[11,12$, 14]. We present an example from elasticity theory in Section 2 to show one application of the SIDE (1)-(2) discussed in this paper.

Since analytic solution to SIDE is rarely available, we look for an approximate solution to the SIDE using direct methods. Most of early direct methods for SIE and SIDE are designed for the case where the boundary $\Gamma$ is a unit circle or an interval [1], [3]-[7], [8, 18]. The requirement of unit circle is essential because some special polynomial interpolations are only defined on the unit circle, see [13], for example. There are few papers that deal with arbitrary closed contours in the literature.

It should be mentioned that using a conforming mapping, one can transform an arbitrary smooth closed contour to the unit circle. However, this approach may not simplify the problem due to the following:

- It is not an easy job to find a conforming mapping.

- The coefficients, the kernels, and the right hand side of the transformed equation may be more complicated and may not be smooth anymore.

- The convergence analysis may be more complicated due to the transformation of the contour.

In the 1980-90's, theoretical foundations for direct methods for SIE and systems of SIE defined on arbitrary smooth contours in the complex plane were established in $[22,23]$, and maybe others. But direct methods for SIDE or system of SIDE for arbitrary contours are yet to be developed and analyzed.

In this paper, we develop a collocation method for the systems of SIDE with arbitrary smooth contours, and the mechanical quadrature rule, see[23] for the definition, to approximate the integrals involved in the system of SIDE. We also give the convergence analysis of our new method in Lebesgue spaces.

The classical continuous function space can not be used because the singular operator of the integration is unbounded. The classical theory of projection methods for $L_{p}$ spaces does not apply because the norm of some projectors (for example, the projectors of interpolation) is unbounded for Lebesgue spaces. Thus, it is necessary to elaborate a new theory for the collocation and quadrature method discussed in this paper. 
The paper is organized as follows. In the next section, we present an application of SIDE to show the importance of SIDE. In Section 3, we describe the numerical schemes for systems of SIDE with an arbitrary smooth contour. The convergence analysis is given in Section 4. Numerical examples are provided in Section 5.

\section{An application of singular integro-differential equations}

We show an application of SIDE in this section to show the importance of our work on SIDE.

Consider an elastic medium under conditions of plane strain or generalized plane stress which fills all of the infinite plane of the variable $z$ and is weakened by a hole of arbitrary shape. By convention, this problem refers to the determination of the stress components along the contour of the hole when external forces are prescribed on the same contour. The state of stress at infinity is given.

As described in [9], the physical model is the following:

$$
\left\{\begin{array}{c}
\sigma_{\rho}^{(1)}+\sigma_{v}^{(1)}=2\left(\Phi_{1}(\xi)+\overline{\Phi_{1}(\xi)}\right) \\
\sigma_{v}^{(1)}-\sigma_{\rho}^{(1)}+2 i \tau_{r v}^{(1)}=\frac{2 \xi^{2}}{\rho^{2} \overline{\omega^{\prime}(\xi)}}\left(\overline{\omega(\xi)} \Phi_{1}^{\prime}(\xi)+\omega^{\prime}(\xi) \Psi_{1}(\xi)\right),
\end{array}\right.
$$

where $\sigma_{\rho}^{(1)}, \sigma_{v}^{(1)}, \tau_{r v}^{(1)}$ are the components of the stress in the curvilinear coordinates related to the conformal mapping

$$
\begin{gathered}
z=\omega(\xi), \quad \xi=\rho e^{i v} \\
\Phi_{1}(\xi)=\frac{\phi_{1}^{\prime}(\xi)}{\omega^{\prime}(\xi)}, \quad \Psi_{1}(\xi)=\frac{\psi_{1}^{\prime}(\xi)}{\omega^{\prime}(\xi)} \\
\phi_{1}(\xi)=\phi(\omega(\xi)), \quad \psi_{1}(\xi)=\psi(\omega(\xi)),
\end{gathered}
$$

and $\phi(z), \psi(z)$, as always, denote the complex potentials in the $z$ plane.

Suppose that the region $\Sigma$ with boundary $\gamma$ which is mapped by relation (4) onto the physical region $S$ represents an infinite region in the plane of the auxiliary variable $\xi$ outside the unit circle centered at the origin. This means that the function $\omega(\xi)$ is representable in the region $\Sigma$ as the series

$$
\omega(\xi)=R \xi+\frac{c_{1}}{\xi}+\frac{c_{2}}{\xi^{2}}+\ldots+\frac{c_{n}}{\xi^{n}} \ldots
$$

It may also be recalled that the functions $\phi_{1}(\xi), \psi_{1}(\xi)$ admit, in the same region the representation,

$$
\left\{\begin{array}{c}
\phi_{1}(\xi)=-\frac{X+i Y}{2 \pi(1+\kappa)} \log \xi+R \Gamma \xi+\phi_{*}(\xi) \\
\psi_{1}(\xi)=-\frac{\kappa(X-i Y)}{2 \pi(1+\kappa)} \log \xi+R \Gamma^{\prime} \xi+\psi_{*}(\xi)
\end{array}\right.
$$


where $\phi_{*}, \psi_{*}$ are homomorphic functions, regular at $\xi=\infty$, and $X, Y$ are the components of the resultant of the external forces applied to the boundary of the region $S$.

Assume now

$$
\sigma_{\rho}^{(1)}=\sigma_{\rho}+\sigma_{\rho}^{0}, \quad \sigma_{v}^{(1)}=\sigma_{v}+\sigma_{v}^{0}, \quad \tau_{r v}^{(1)}=\tau_{r v}+\tau_{r v}^{0},
$$

where $\sigma_{\rho}^{0}, \sigma_{v}^{0}, \tau_{r v}^{0}$ are the components of stress in a uniform field characterized by the constants $\Gamma$ and $\Gamma^{\prime}(\Gamma=\bar{\Gamma})$, and represent relations $(3)$ with $\rho=1$ as follows $\left(\sigma=e^{i v}\right)$ :

$$
\begin{aligned}
2(\Phi(\sigma)+\overline{\Phi(\sigma)}) & =\sigma_{\rho}+\sigma_{v} \text { on } \gamma \\
2\left(\overline{\omega(\sigma)} \Phi^{\prime}(\sigma)+\omega^{\prime}(\sigma) \Psi(\sigma)\right) & =\overline{\sigma^{2} \omega^{\prime}(\sigma)}\left(\sigma_{v}-\sigma_{\rho}+2 i \tau_{\rho v}\right) .
\end{aligned}
$$

Here $\Phi$ and $\Psi$ are two unknown functions, homomorphic in the region $\Sigma$. From (5) to (7), we have the following asymptotic when $|\xi|$ is large,

$$
\begin{gathered}
\Phi(\xi)=\frac{A}{\xi}+O\left(\xi^{-2}\right), \quad \Psi(\xi)=\frac{A^{\prime}}{\xi}+O\left(\xi^{-2}\right), \\
A=-\frac{X+i Y}{2 \pi R(1+\kappa)}, \quad A^{\prime}=\frac{\kappa(X-i Y)}{2 \pi R(1+\kappa)} .
\end{gathered}
$$

The stresses $\sigma_{\rho}^{0}, \sigma_{v}^{0}, \tau_{\rho v}^{0}$ then can be calculated from

$$
\left\{\begin{array}{c}
\sigma_{\rho}^{0}+\sigma_{v}^{0}=2\left(\Phi_{0}(\xi)+\overline{\Phi_{0}(\xi)}\right) \\
\sigma_{v}^{0}-\sigma_{\rho}^{0}+2 i \tau_{\rho v}^{0}=\frac{2 \xi^{2}}{\rho^{2} \overline{\omega^{\prime}(\xi)}}\left(\overline{\omega(\xi)} \Phi_{0}^{\prime}(\xi)+\omega^{\prime}(\xi) \Psi_{0}(\xi)\right)
\end{array}\right.
$$

with

$$
\Phi_{0}=\frac{R \Gamma}{\omega^{\prime}(\xi)}, \quad \Psi_{0}(\xi)=\frac{R \Gamma^{\prime}}{\omega^{\prime}(\xi)} .
$$

Compared with (8), we have

$$
\Phi_{1}(\xi)=\Phi(\xi)+\Phi_{0}(\xi), \quad \Psi_{1}(\xi)=\Psi(\xi)+\Psi_{0}(\xi)
$$

It is well known that, whenever the region occupied by the elastic medium contains the infinitely remote point of the plane, the constants $\Gamma, \Gamma^{\prime}, X, Y$ are specified by the problem itself. The stresses $\sigma_{\rho}^{0}, \sigma_{v}^{0}, \tau_{r v}^{0}$ and the constants $A$ and $A^{\prime}$ are therefore known, they are defined by formulas (11) and (12).

Our problem is to determine the normal stress $\sigma_{v}$ by formulas (9) from the given values on circumference $\gamma$ of the other two components, $\sigma_{\rho}$ and $\tau_{\rho v}$. Assume

$$
\sigma_{v}+\sigma_{\rho}=\Omega(\sigma), \quad-2\left(\sigma_{\rho}-i \tau_{\rho v}\right)=\Theta(\sigma) \quad \text { on } \quad \gamma
$$


and rewrite (9) as

$$
\left\{\begin{array}{c}
2(\Phi(\sigma)+\overline{\Phi(\sigma)})=\Omega(\sigma) \\
\overline{\sigma^{2} \omega^{\prime}(\sigma)}(\Omega(\sigma)+\Theta(\sigma))-2 \overline{\omega(\sigma)} \Phi^{\prime}(\sigma)=2 \omega^{\prime}(\sigma) \Psi(\sigma) \quad \text { on } \quad \gamma
\end{array}\right.
$$

After the transformations we obtain the required equation for determining $\Omega(\sigma)$ as follows:

$$
\begin{gathered}
\overline{\sigma^{2} \omega^{\prime}(\sigma)}[\Omega(\sigma)+\Theta(\sigma)]+\frac{1}{\pi i} \int_{\gamma} \overline{\tau^{2} \omega^{\prime}(\tau)}[\Omega(\tau)+\Theta(\tau)] \frac{d \tau}{\tau-\sigma}- \\
-\frac{1}{\pi i} \int_{\gamma} \frac{\overline{\omega(\tau)}-\overline{\omega(\sigma)}}{\tau-\sigma} \Omega^{\prime}(\tau) d \tau-\frac{4 \bar{A} R}{\sigma}=0 \quad \text { on } \gamma,
\end{gathered}
$$

where $\bar{A}, R, \Theta(\sigma)$ are known. After some manipulation, see [10] as well, we obtain

$$
\begin{aligned}
& \overline{\sigma^{2} \omega^{\prime}(\sigma)} \Omega(\sigma)+\frac{\overline{\sigma^{2} \omega^{\prime}(\sigma)}}{\pi i} \int_{\gamma} \frac{\Omega(\tau)}{\tau-\sigma} d \tau \\
& -\frac{1}{\pi i} \int_{\gamma} \frac{\overline{\omega(\tau)}-\overline{\omega(\sigma)}}{\tau-\sigma} \Omega^{\prime}(\tau) d \tau+\frac{1}{\pi i} \int_{\gamma} \frac{\overline{\tau^{2} \omega^{\prime}(\tau)}-\overline{\sigma^{2} \omega^{\prime}(\sigma)}}{\tau-\sigma} \Omega(\tau) d \tau \\
& =\frac{4 \bar{A} R}{\sigma}-\overline{\sigma^{2} \omega^{\prime}(\sigma)} \Theta(\sigma)-\frac{1}{\pi i} \int_{\gamma} \frac{\overline{\tau^{2} \omega^{\prime}(\tau)} \Theta(\tau)}{\tau-\sigma} d \tau
\end{aligned}
$$

or

$$
A(\sigma) \Omega(\sigma)+\frac{A(\sigma)}{\pi i} \int_{\gamma} \frac{\Omega(\tau)}{\tau-\sigma} d \tau+\frac{1}{\pi i} \sum_{\nu=0}^{1} \int_{\gamma} K_{\nu}(\sigma, \tau) \Omega^{(\nu)}(\tau) d \tau=f(\sigma)
$$

where

$$
\begin{gathered}
A(\sigma)=\overline{\sigma^{2} \omega^{\prime}(\sigma)}, \quad K_{0}(\sigma, \tau)=\frac{\overline{\tau^{2} \omega^{\prime}(\tau)}-\overline{\sigma^{2} \omega^{\prime}(\sigma)}}{\tau-\sigma}, \quad K_{1}(\sigma, \tau)=\frac{\overline{\omega(\sigma)}-\overline{\omega(\tau)}}{\tau-\sigma} \\
f(\sigma)=\frac{4 \bar{A} R}{\sigma}-\overline{\sigma^{2} \omega^{\prime}(\sigma)} \Theta(\sigma)-\frac{1}{\pi i} \int_{\gamma} \frac{\overline{\tau^{2} \omega^{\prime}(\tau)} \Theta(\tau)}{\tau-\sigma} d \tau .
\end{gathered}
$$

If $\omega(\sigma), f(\sigma), A(\sigma)$ are Hölder functions on $\gamma$, then $K_{\nu}(\sigma, \tau)=\frac{\tilde{K}_{\nu}(\sigma, \tau)}{|\tau-\sigma|^{\lambda}}$, and $\tilde{K}_{\nu}(\sigma, \tau)$, are also Hölder functions for $0 \leq \lambda<1$, and $\nu=\overline{0,1}$, see [10]. Thus the application problem can be described by the SIDE (1)-(2) with $\lambda=0$.

\section{A collocation and quadrature method for system of SIDE}

Before we describe our numerical schemes, we need some theoretical preparations. 


\subsection{Theoretical preparations}

Let $\Gamma$ be a smooth Jordan curve which divides the entire complex plane $C$ into two parts, $F^{-}$and $F^{+}$. Assume that $F^{-}$contains the infinity and $F^{+}$contains the origin. Therefore $F^{-}=C-\left\{F^{+} \cup \Gamma\right\}$. Let $z=\psi(w)$ be an analytic function that maps conformally the exterior of the unit circle to $F^{-}$so that $\psi(\infty)=\infty, \psi^{(\prime)}(\infty)>0$.

Let us consider the Riemann function $t=\psi(w), w \in \Gamma_{0}=\{\xi:|\xi|=1\}$, that has at least up to second derivatives. Assume that $\psi^{(2)}(w)$ satisfies the Hölder condition on $|w|=1$, that is $\psi^{(2)}(w) \in H_{\mu}\left(\Gamma_{0}\right)$, for some parameter $\mu, \mu(0<\mu<1)$. We denote the set of such contours by $C(2 ; \mu)$, see [23] for more details.

In the complex $\left[L_{p}(\Gamma)\right]_{m}(1<p<\infty)$ space, the norm of a vector functions $g(t)=$ $\left(g_{1}(t), \ldots, g_{m}(t)\right)$ is defined as,

$$
\|g\|=\sum_{k=1}^{m}\left\|g_{k}\right\|_{p} ; \quad\left\|g_{k}\right\|_{p}=\left(\frac{1}{l} \int_{\Gamma}\left|g_{k}\right|^{p}|d \tau|\right)^{\frac{1}{p}},
$$

where $l$ is the length of $\Gamma$.

We will develop a direct method for the systems of SIDE (1) together with conditions (2).

\subsection{A collocation and quadrature method}

Using the operators $P=\frac{1}{2}(I+S), Q=I-P$, where $I$ is the identity operator, and $S$ is a singular Cauchy nucleus, equation (1) can be written as follows:

$$
\begin{aligned}
(M x & \equiv) \sum_{r=0}^{\nu}\left(A_{r}(t)\left(P x^{(r)}\right)(t)+B_{r}(t)\left(Q x^{(r)}\right)(t)+\right. \\
& \left.+\frac{1}{2 \pi i} \int_{\Gamma} K_{r}(t, \tau) x^{(r)}(\tau) d \tau\right)=f(t), \quad t \in \Gamma
\end{aligned}
$$

where $A_{r}(t)=\tilde{A}_{r}(t)+\tilde{B}_{r}(t), B_{r}(t)=\tilde{A}_{r}(t)-\tilde{B}_{r}(t), \quad r=\overline{0, \nu}$.

We seek an approximate solution to problem (1)-(2) of the form

$$
x_{n}(t)=\sum_{k=0}^{n} \xi_{k}^{(n)} t^{k+\nu}+\sum_{k=-n}^{-1} \xi_{k}^{(n)} t^{k}, \quad t \in \Gamma,
$$

where $\xi_{k}^{(n)}=\xi_{k}, k=\overline{-n, n}$ are unknown vectors of dimension $m$. Note that $x_{n}(t)$ 's constructed using (19) satisfy the condition (2). 
In our collocation method, we choose $2 n+1$ different points on $\Gamma\left\{t_{j}\right\}$ such that the SIDE can be satisfied exactly

$$
\left(M x_{n}\right)\left(t_{j}\right)-f\left(t_{j}\right)=0,
$$

As a result, we obtain a system of linear algebraic equations for the coefficients $\xi_{k}$, $k=\overline{-n, n}$,

$$
\begin{aligned}
\sum_{r=0}^{\nu}\left\{A_{r}\left(t_{j}\right) \sum_{k=0}^{n} \frac{(k+\nu) !}{(k+\nu-r) !} t^{k+\nu-r} \xi_{k}+B_{r}\left(t_{j}\right) \sum_{k=1}^{n}(-1)^{r} \frac{(k+r-1) !}{(k-1) !}\right. \\
\cdot t_{j}^{-k-r} \cdot \xi_{-k}+\frac{1}{2 \pi i} \cdot \sum_{k=0}^{n} \frac{(k+\nu) !}{(k+\nu-r) !} \int_{\Gamma} K_{r}\left(t_{j}, \tau\right) \tau^{k+\nu-r} d \tau \cdot \xi_{k} \\
\left.+\sum_{k=1}^{n}(-1)^{r} \frac{(k+r-1) !}{(k-1) !} \cdot \frac{1}{2 \pi i} \int_{\Gamma} K_{r}\left(t_{j}, \tau\right) \tau^{-k-r} d \tau \cdot \xi_{-k}\right\}=f\left(t_{j}\right),
\end{aligned}
$$

where $j=\overline{0,2 n}$.

We need to approximate the integrals in the system of linear algebraic equations above as well. This is done using the mechanical quadrature formulae described in [23],

$$
\frac{1}{2 \pi i} \int_{\Gamma} g(\tau) \tau^{l+k} d \tau \approx \frac{1}{2 \pi i} \int_{\Gamma} U_{n}\left(\tau^{l+1} \cdot g(\tau)\right) \tau^{k-1} d \tau,
$$

where the operator of interpolation $U_{n}$ is determined by, see [23],

$$
\begin{gathered}
\left(U_{n} g\right)(t)=\sum_{s=0}^{2 n} g\left(t_{s}\right) \cdot l_{s}(t), \\
l_{j}(t)=\prod_{k=0, k \neq j}^{2 n} \frac{t-t_{k}}{t_{j}-t_{k}}\left(\frac{t_{j}}{t}\right)^{n} \equiv \sum_{k=-n}^{n} \Lambda_{k}^{(j)} t^{k}, \quad t \in \Gamma, \quad j=\overline{0,2 n} .
\end{gathered}
$$

Thus, the system of linear algebraic equations for the unknown $\xi_{k}$ of (21) has been approximated by the following system of linear algebraic equations

$$
\begin{aligned}
\sum_{r=0}^{\nu}\{ & A_{r}\left(t_{j}\right) \sum_{k=0}^{n} \frac{(k+\nu) !}{(k+\nu-r) !} t_{j}^{k+\nu-r} \xi_{k} \\
& +B_{r}\left(t_{j}\right) \sum_{k=1}^{n}(-1)^{r} \frac{(k+r-1) !}{(k-1) !} t_{j}^{-k-r} \cdot \xi_{-k} \\
& +\sum_{k=0}^{n} \frac{(k+\nu) !}{(k+\nu-r) !} \sum_{s=0}^{2 n} K_{r}\left(t_{j}, t_{s}\right) t_{s}^{1+\nu-r} \Lambda_{-k}^{(s)} \xi_{k} \\
& \left.+\sum_{k=1}^{n}(-1)^{r} \frac{(k+r-1) !}{(k-1) !} \sum_{s=0}^{2 n} K_{r}\left(t_{j}, t_{s}\right) t_{s}^{-1-r} \Lambda_{k}^{(s)} \xi_{-k}\right\}=f\left(t_{j}\right)
\end{aligned}
$$

for $j=\overline{0,2 n}$. Thus we have obtained the system of linear algebraic equations for the unknowns $\xi_{k}$ in (19). Once these coefficients are obtained, the solution to the singular integro-differential equations is expressed as (19). 


\section{Error estimates for the numerical method}

In this section, we give some error estimates for the numerical method proposed in the previous section.

As was proved in [10], a paired vector functions $\frac{d^{\nu}(P x)(t)}{d t^{\nu}}$ and $\frac{d^{\nu}(Q x)(t)}{d t^{\nu}}$ can be represented by the integrals of Cauchy type with the same density $\zeta(t)$ :

$$
\left.\begin{array}{cl}
\frac{d^{\nu}(P x)(t)}{d t^{\nu}}=\frac{1}{2 \pi i} \int_{\Gamma} \frac{\zeta(\tau)}{\tau-t} d \tau, & t \in F^{+} \\
\frac{d^{\nu}(Q x)(t)}{d t^{\nu}}=\frac{t^{-\nu}}{2 \pi i} \int_{\Gamma} \frac{\zeta(\tau)}{\tau-t} d \tau, & t \in F^{-} .
\end{array}\right\}
$$

By means of the above formulae, the problem (1)-(2) can be reduced to an equivalent (in terms of solving) singular integral equations system

$$
(R \zeta \equiv) C(t) \zeta(t)+\frac{D(t)}{\pi i} \int_{\Gamma} \frac{\zeta(\tau)}{\tau-t} d \tau+\frac{1}{2 \pi i} \int_{\Gamma} h(t, \tau) \zeta(\tau) d \tau=f(t), \quad t \in \Gamma,
$$

where

$$
\begin{aligned}
C(t) & =\frac{1}{2}\left[A_{\nu}(t)+t^{-\nu} B_{\nu}(t)\right], \\
D(t) & =\frac{1}{2}\left[A_{\nu}(t)-t^{-\nu} B_{\nu}(t)\right],
\end{aligned}
$$

and $h(t, \tau)$ is a matrix function belonging to $[C(\Gamma)]_{m}$ space for both variables $t$ and $\tau$, see [10]. The equivalence of the existence of the solution between the system of SIE (25) and the problem (1)-(2) is the result of the following lemma from [10].

Lemma 1 The system of SIE (25) and the problem (1)-(2) are equivalent in terms of the solvability. That is, for each solution $\zeta(t)$ of system (25), there is a solution $x(t)$ of the problem (1)-(2), determined by the following formulae

$$
\begin{aligned}
& (P x)(t)=\frac{(-1)^{\nu}}{2 \pi i(\nu-1) !} \int_{\Gamma} \zeta(\tau)\left((\tau-t)^{\nu-1} \log \left(1-\frac{t}{\tau}\right)+\sum_{k=1}^{\nu-1} \tilde{\alpha}_{k} \tau^{\nu-k-1} t^{k}\right) d \tau \\
& (Q x)(t)=\frac{(-1)^{\nu}}{2 \pi i(\nu-1) !} \int_{\Gamma} \zeta(\tau) \tau^{-\nu}\left((\tau-t)^{\nu-1} \log \left(1-\frac{\tau}{t}\right)+\sum_{k=1}^{\nu-2} \tilde{\beta}_{k} \tau^{\nu-k-1} t^{k}\right) d \tau
\end{aligned}
$$

where $\tilde{\alpha}_{k}$ and $\tilde{\beta}_{k}$, are real numbers. On the other hand, for each solution $x(t)$ of problem (1)-(2), there is a solution $\zeta(t)$

$$
\zeta(t)=\frac{d^{\nu}(P x)(t)}{d t^{\nu}}+t^{\nu} \frac{d^{\nu}(Q x)(t)}{d t^{\nu}}
$$

to the system (25). Furthermore, given a set of linear-independent solutions $\zeta(t)$ to the system (25), there are corresponding set of linear-independent solutions to the problem (1)-(2) from (27) and vise versa. 
The proof can be found in [10].

Before we discuss the main convergence theorem of our method, we need several additional lemmas from [19]. Those lemmas will be used in our discussions in this section.

Define the space

$$
\left[\stackrel{\circ}{W}_{p}^{(\nu)}\right]_{m}(\Gamma)=\left\{g ; \exists g^{(r)} \in[C(\Gamma)]_{m}, r=\overline{0, \nu-1}, g^{(\nu)} \in\left[L_{p}(\Gamma)\right]_{m}\right\} .
$$

For any $g \in\left[\stackrel{\circ}{{ }^{\circ}} p\right]_{m}, g$ satisfies equation (2). The norm in $\left[\stackrel{\circ}{W}_{p}^{(\nu)}\right]_{m}$ is defined by

$$
\|g\|_{p, \nu}=\left\|g^{(\nu)}\right\|_{\left[L_{p}\right]_{m}} .
$$

We shall denote the image of space $\left[L_{p}\right]_{m}$ under the mapping $P+t^{-\nu} Q$ by $\left[L_{p, \nu}\right]_{m}$ with the same norm as in $\left[L_{p}\right]_{m}$.

Lemma 2 The differential operator $D^{\nu}:\left[\stackrel{\circ}{W_{p}^{(\nu)}}\right]_{m} \rightarrow\left[L_{p, \nu}\right]_{m},\left(D^{\nu} g\right)(t)=g^{(\nu)}(t)$ is continuously reversible and its reverse operator $D^{-\nu}:\left[L_{p, \nu}\right]_{m} \rightarrow\left[\stackrel{\circ}{W}_{p}^{(\nu)}\right]_{m}$ is determined by the equality

$$
\left(D^{-\nu} g\right)(t)=\left(N^{+} g\right)(t)+\left(N^{-} g\right)(t)
$$

where

$$
\begin{aligned}
& \left(N^{+} g\right)(t)=\frac{(-1)^{\nu}}{2 \pi i(\nu-1) !} \int_{\Gamma}(P g)(\tau)(\tau-t)^{\nu-1} \log \left(1-\frac{t}{\tau}\right) d \tau \\
& \left(N^{-} g\right)(t)=\frac{(-1)^{\nu-1}}{2 \pi i(\nu-1) !} \int_{\Gamma}(Q g)(\tau)(\tau-t)^{\nu-1} \log \left(1-\frac{\tau}{t}\right) d \tau .
\end{aligned}
$$

The proof can be found in [19].

Lemma 3 The operator $B:\left[\stackrel{\circ}{W_{p}^{(\nu)}}\right]_{m} \rightarrow\left[L_{p}\right]_{m}$, of the form $B=\left(P+t^{\nu} Q\right) D^{\nu}$ is reversible and

$$
B^{-1}=D^{-\nu}\left(P+t^{-\nu} Q\right) .
$$

The proof can also be found in [19].

The existence of the solution to the systems of linear algebraic equations (21) and (23) is given in the following theorems.

Theorem 4 Assume the following conditions are satisfied:

1. the contour $\Gamma \in C(2, \mu), \quad 0<\mu<1$, 
2. the matrix functions $A_{r}(t)$ and $B_{r}(t)$ belong to the space $\left[H_{\alpha}(\Gamma)\right]_{m}, 0<\alpha<1$, for $r=\overline{0, \nu}$,

3. $\operatorname{det}\left(A_{\nu}(t)\right) \cdot \operatorname{det}\left(B_{\nu}(t)\right) \neq 0$, for any $t \in \Gamma$,

4. the left partial indexes of $t^{\nu} B_{\nu}^{-1}(t) A_{\nu}(t)$ are zero, for any $t \in \Gamma$,

5. the matrix functions $K_{r}(t, \tau) \in H_{\beta}[(\Gamma)]_{m}, \quad 0<\beta \leq 1$ with variables $t$ and $\tau$, and the vector function $f(t) \in[C(\Gamma)]_{m}$ for any $t \in \Gamma$,

6. the operator $M:\left[\stackrel{\circ}{W}_{p}^{(\nu)}\right]_{m} \rightarrow\left[L_{p}(\Gamma)\right]_{m}$ is linearly reversible,

7. the points $t_{j}(j=\overline{0,2 n})$ form a system of Feyer knots, see [21] for the definition, on $\Gamma$ :

$$
t_{j}=\psi\left(\exp \left(\frac{2 \pi i}{2 n+1}(j-n)\right)\right), \quad j=\overline{0,2 n}, \quad i^{2}=-1 .
$$

Then, if $n$ is large enough, the system of linear equations (21) has a unique solution $\xi_{k}$, $k=\overline{-n, n}$. The approximate solutions $x_{n}(t)$, constructed from formula (19), converges as $n \rightarrow \infty$ in the normed space $\left[\stackrel{\circ}{{ }^{\circ}}{ }_{p}^{(\nu)}\right]_{m}$ to the exact solution $x(t)$ of SIDE (1)-(2). Furthermore, the following error estimate holds:

$$
\left\|x-x_{n}\right\|_{p, \nu} \leq O\left(\frac{1}{n^{\alpha}}\right)+O\left(\omega\left(f ; \frac{1}{n}\right)\right)+O\left(\omega^{t}\left(h ; \frac{1}{n}\right)\right) \stackrel{\text { def }}{=} \delta_{n} .
$$

Proof: We show that if $n$ is large enough, then the operator $U_{n} M U_{n}$ is reversible. The operator acts on the subspace

$$
\left[\stackrel{\circ}{X}_{n}\right]_{m}=\left\{t^{\nu} \sum_{k=0}^{n} \alpha_{k} t^{k}+\sum_{k=-n}^{-1} \alpha_{k} t^{k}\right\}
$$

with the norm defined in $\left[\stackrel{\circ}{W_{p}^{(\nu)}}\right]_{m}$ to the subspace

$$
\left[R_{n}\right]_{m}=\left\{\sum_{k=-n}^{n} r_{k} t^{k}\right\}, \quad t \in \Gamma,
$$

with the norm defined in $\left[L_{p}(\Gamma)\right]_{m}$.

Similarly to (24) we represent $\frac{d^{\nu}\left(P x_{n}\right)(t)}{d t^{\nu}}$ and $\frac{d^{\nu}\left(Q x_{n}\right)(t)}{d t^{\nu}}$ by integrals of Cauchy type with the same density $\zeta_{n}(t)$ :

$$
\left.\begin{array}{cc}
\frac{d^{\nu}\left(P x_{n}\right)(t)}{d t^{\nu}}=\frac{1}{2 \pi i} \int_{\Gamma} \frac{\zeta_{n}(\tau)}{\tau-t} d \tau, & t \in F^{+} \\
\frac{d^{\nu}\left(Q x_{n}\right)(t)}{d t^{\nu}}=\frac{t^{-\nu}}{2 \pi i} \int_{\Gamma} \frac{\zeta_{n}(\tau)}{\tau-t} d \tau, & t \in F^{-} .
\end{array}\right\}
$$


It is easy to prove that

$$
\zeta_{n}(t)=\sum_{k=0}^{n} \frac{(k+\nu) !}{k !} t^{k} \xi_{k}+(-1)^{\nu} \sum_{k=1}^{n} \frac{(k+\nu-1) !}{(k-1) !} t^{-k} \xi_{-k}
$$

and therefore $\zeta_{n}(t) \in\left[R_{n}\right]_{m}$, for all $t \in \Gamma$.

Using (30), we reduce the equation $U_{n} M U_{n} x_{n}=U_{n} f$, to an equivalent equation, in terms of solvability,

$$
U_{n} R U_{n} x_{n}=U_{n} f
$$

in the subspaces $\left[R_{n}\right]_{m}$.

Obviously, the last equation, represents a discrete system of equations of the collocation method for the system of SIE (25). From (30) and the fact that $\zeta_{n} \in\left[R_{n}\right]_{m}$, we conclude that if $\zeta_{n}(t)$ is the solution of the equation (31), then $y_{n}(t)$, defined by

$$
\begin{aligned}
& \left(P y_{n}\right)(t)=\frac{(-1)^{\nu}}{2 \pi i(\nu-1) !} \int_{\Gamma} \zeta_{n}(\tau)\left\{(\tau-t)^{\nu-1} \log \left(1-\frac{t}{\tau}\right)+\sum_{k=1}^{\nu-1} \tilde{\alpha}_{k} \tau^{\nu-k-1} t^{k}\right\} d \tau \\
& \left(Q y_{n}\right)(t)=\frac{(-1)^{\nu}}{2 \pi i(\nu-1) !} \int_{\Gamma} \zeta_{n}(\tau) \tau^{-\nu}\left\{(\tau-t)^{\nu-1} \log \left(1-\frac{\tau}{t}\right)+\sum_{k=1}^{\nu-2} \tilde{\beta}_{k} \tau^{\nu-k-1} t^{k}\right\} d \tau
\end{aligned}
$$

is the discrete solution of the system $U_{n} M U_{n} x_{n}=U_{n} f$ and vice versa. As we have mentioned earlier, the vector functions $y_{n}(t)$ are determined uniquely in terms of $\zeta_{n}(t)$ according to formulae (32).

It follows that if the equation (31) has a unique solution $\zeta_{n}(t)$ in the subspace $\left[R_{n}\right]_{m}$, then the equality $y_{n}(t)=x_{n}(t)$ is true. Therefore $x_{n}(t)$ has been uniquely determined.

The discrete system of equation (31) is obtained from the collocation method applied to (25). We will show that for this system, all conditions of Theorem 8.3 from [23] are satisfied. From the condition 3 of Theorem 4 and formula (26), we have $\operatorname{det}[C(t) \pm D(t)] \neq 0$ for any $t \in \Gamma$, which coincides with the condition 3 of Theorem 8.3 from [23]. From the equality

$$
[C(t)-D(t)]^{-1}[C(t)+D(t)]=t^{\nu} B_{\nu}^{-1}(t) A_{\nu}(t)
$$

and the condition 4 of Theorem 4, we conclude the left partial indexes matrix functions of $[C(t)-D(t)]^{-1}[C(t)+D(t)]$ has to be zero. This is the condition 4 of Theorem 8.3 in $[23]$.

From Lemma 3 and the conditions 3, 4, 6 listed in Theorem 4, the reversibility of operator $R:\left[L_{p}(\Gamma)\right]_{m} \rightarrow\left[L_{p}(\Gamma)\right]_{m}$ follows. Note that the conditions 1, 2, 5 , and 7, coincide with the conditions for Theorem 8.3 from [23]. Therefore, if $n$ is large enough, the system (31) has a unique solution $\zeta_{n}(t) \in\left[R_{n}\right]_{m}$. Hence, the linear system $U_{n} M U_{n} x_{n}=U_{n} f$, and the system of linear algebraic equations (21) has a unique solution if $n$ is large enough. 
From Theorem 8.3 in [23], we know that

$$
\left\|\zeta-\zeta_{n}\right\|_{\left[L_{p}\right]_{m}} \leq O\left(\frac{1}{n^{\alpha}}\right)+O\left(\omega\left(f ; \frac{1}{n}\right)\right)+O\left(\omega^{t}\left(h ; \frac{1}{n}\right)\right) .
$$

From (24) and (30) we obtain

$$
(P x)^{(\nu)}(t)=(P \zeta)(t) \text { and }(Q x)^{(\nu)}(t)=t^{-\nu} \cdot(Q \zeta)(t) .
$$

Therefore we have

$$
\begin{aligned}
\left(P x_{n}\right)^{(\nu)}(t) & =\left(P \zeta_{n}\right)(t), \\
\left(Q x_{n}\right)^{(\nu)} & =t^{-\nu}\left(Q \zeta_{n}\right)(t) .
\end{aligned}
$$

We proceed to get an error estimate

$$
\begin{aligned}
\left\|x-x_{n}\right\|_{p, \nu} & =\left\|x^{(\nu)}-x_{n}^{(\nu)}\right\|_{\left[L_{p}\right]_{m}} \\
& \leq\left\|P\left(\zeta-\zeta_{n}\right)\right\|_{\left[L_{p}\right]_{m}}+\left\|t^{-\nu} Q\left(\zeta-\zeta_{n}\right)\right\|_{\left[L_{p}\right]_{m}} \\
& \leq\|P\| \cdot\left\|\zeta-\zeta_{n}\right\|_{\left[L_{p}\right]_{m}}+\left\|t^{-\nu}\right\|_{L_{p}} \cdot\|Q\| \cdot\left\|\zeta-\zeta_{n}\right\|_{\left[L_{p}\right]_{m}} \\
& \leq\left(\|P\|+\left\|t^{-\nu}\right\| \cdot\|Q\|\right)\left\|\zeta-\zeta_{n}\right\| \\
& =\left(\|P\|+c_{1}\|Q\|\right)\left\|\zeta-\zeta_{n}\right\| .
\end{aligned}
$$

Here we have used the inequality

$$
\begin{aligned}
\left\|t^{-\nu}\right\|_{L_{p}} & =\left(\frac{1}{l} \int_{\Gamma}\left|t^{-\nu}\right|^{p} d t\right)^{\frac{1}{p}}=\left(\frac{1}{l} \int_{\Gamma}\left|t^{-p \nu}\right| d t\right)^{\frac{1}{p}} \\
& \leq\left(\frac{1}{l} \cdot \frac{1}{\min _{t \in \Gamma}|t|^{p \cdot \nu}} \cdot l\right)^{\frac{1}{p}}=\left(\frac{1}{\min _{t \in \Gamma}|t|^{p \cdot \nu}}\right)^{\frac{1}{p}}=c_{1} .
\end{aligned}
$$

From the previous inequality (34) and with the help of (33) we obtain (29). That completes the proof of the theorem.

The result in the convergence theorem assume that the integrations in the system of linear algebraic equations (21) can be calculated exactly. However, in practice, those integrations are evaluated using the mechanical quadrature rule. We introduce the following convergence theorem if the integrations are approximated by the mechanical quadrature rule [23].

Theorem 5 Assume that all the conditions 1-7 in Theorem 4 are satisfied. Then, if $n$ is large enough, the system of linear algebraic equations (23) has a unique solution $\xi_{k}, k=\overline{-n, n}$. The approximate solution of (19) converges to the exact solution $x(t)$ 
of SIDE (1)-(2) in the norm $\left[\stackrel{\circ}{[}_{p}^{(\nu)}\right]_{m}$ as $n \rightarrow \infty$. Furthermore, the following error estimate is true,

$$
\left\|x-x_{n}\right\|_{p, \nu}=\delta_{n}+O\left(\omega^{\tau}\left(h ; \frac{1}{n}\right)\right)+O\left(\frac{\log (2 n+1)}{n^{\alpha}}\right) .
$$

Proof: It is easy to verify that the system of singular integro-differential equations (23) is equivalent to the operator equation

$$
\begin{aligned}
U_{n} & \left\{\sum _ { r = 0 } ^ { \nu } \left[A_{r}(t)\left(P x_{n}^{(r)}\right)(t)+B_{r}(t)\left(Q x_{n}^{(r)}\right)(t)\right.\right. \\
& +\frac{1}{2 \pi i} \int_{\Gamma} \frac{1}{\tau} U_{n}^{(\tau)}\left[\tau^{\nu+1-r} K(t, \tau)\right]\left(P x_{n}^{(r)}\right)(\tau) d \tau \\
& \left.\left.+\frac{1}{2 \pi i} \int_{\Gamma} \frac{1}{\tau} U_{n}^{(\tau)}\left[\tau^{-r-1} K(t, \tau)\right]\left(Q x_{n}^{(r)}\right)(\tau) d \tau\right]\right\}=U_{n} f,
\end{aligned}
$$

which is equivalent to the equation, in terms of solvability,

$$
U_{n}\left\{C(t) \zeta_{n}(t)+D(t)\left(S \zeta_{n}\right)(t)+\frac{1}{2 \pi i} \int_{\Gamma} \frac{1}{\tau} U_{n}^{(\tau)}[\tau h(t, \tau)] \cdot \zeta_{n}(\tau) d \tau\right\}=U_{n} f
$$

after the integral representation (30) has been applied. The matrix-functions $C(t)$, $D(t)$, and $h(t, \tau)$ are determined from $(26)$.

The equation (37) represents the system of equations obtained by approximating the integrals in the system of SIE (25) with the mechanical quadrature rule.

From the conditions of Theorem 5, and the property of the mechanical quadrature rule applied to SIE (25), it is easy to verify that (as it has been shown in the proof process of Theorem 4) the conditions of Theorem 8.4 in [23] are satisfied. Therefore, from Theorem 8.4 in [23], we can conclude that the system (37) has a unique solution $\zeta_{n}(t) \in\left[R_{n}\right]_{m}$ if $n$ is large enough. Furthermore, we have the following error estimate,

$$
\begin{aligned}
\| \zeta & -\zeta_{n} \|_{\left[L_{p}\right]_{m}} \leq O\left(\frac{\log (2 n+1)}{n^{\alpha}}\right)+O\left(\frac{1}{n^{\alpha}}\right)+O\left(\omega\left(f ; \frac{1}{n}\right)\right) \\
& +O\left(\omega^{\tau}\left(h ; \frac{1}{n}\right)\right)+O\left(\omega^{t}\left(h ; \frac{1}{n}\right)\right) .
\end{aligned}
$$

For such an $n$, the equation (36) has a unique solution $x_{n}(t) \in\left[\stackrel{\circ}{X}_{n}\right]_{m}$, which is connected with $\zeta_{n}(t)$ by the formulae (32). Here we have used the fact that $x_{n}(t)=y_{n}(t)$. Since the exact solutions $x(t)$ of SIDE (1)-(2) and $\zeta(t)$ are connected by the formulae (27), using the inequality (38), we conclude the equality (35). Thus we have completed the proof for Theorem 5 .

Note that similar results for the SIDE (1)-(2) have been provided in Hölder spaces, for example, see $([24])$. But our results are for Lebesgue spaces. 


\section{A numerical example}

We present the result of an example for $m=1$ to show the performance of our method. In this example, we take the exact solution as

$$
x(t)=\frac{1}{t-1} .
$$

The coefficient matrix functions are chosen as follows

$$
\begin{aligned}
& \tilde{A}_{0}(t)=\tilde{A}_{1}(t)=\frac{1}{2}\left(t+\frac{1}{2}-\frac{1}{t}\right)\left(\frac{1}{t}+1\right), \\
& \tilde{B}_{0}(t)=\tilde{B}_{1}(t)=\frac{1}{2}\left(t+\frac{1}{2}-\frac{1}{t}\right)\left(\frac{1}{t}-1\right), \\
& K_{r}(t, \tau)=\frac{t+r+1}{\tau}, \quad r=\overline{0,1} .
\end{aligned}
$$

The contour $\Gamma$ is an ellipse $R \cos (\phi)+i r \sin (\phi)$. For this example, $R=3$ and $r=2$. The right hand side $f(t)$ then is determined from (1).

In Table 1 and Figure 5, we show the result using the numerical method developed in this paper. The collocation points are taken according to the formula (28).

\begin{tabular}{||c|c||}
\hline \hline $2 \mathrm{n}$ & Error \\
\hline 8 & 0.0749 \\
\hline 16 & 0.0215 \\
\hline 24 & 0.0012 \\
\hline 28 & $2.8018 \mathrm{e}-004$ \\
\hline 32 & $6.4508 \mathrm{e}-005$ \\
\hline \hline
\end{tabular}

Table 1: Error between the exact solution $x(z)$ and the approximate solution $x_{n}(z)$ at selected points $z$ for different $n$. The error is the largest error in the magnitude of all selected points.

In our test, the non-collocation points have been obtained from formula

$$
z(j)=R \cos \left(\frac{2 \pi(j-1)}{k}+\frac{\pi}{16}\right)+r i \sin \left(\frac{2 \pi(j-1)}{k}+\frac{\pi}{16}\right), \quad j=\overline{1, k+1},
$$

where $k$ is a natural number. We have observed that, we should take enough collocation points to guarantee the convergence.

\section{Conclusions}

In this paper, we have proposed a numerical scheme for solving some system of singular integro-differential equations for arbitrary smooth contours in complex plane. The 
(a)

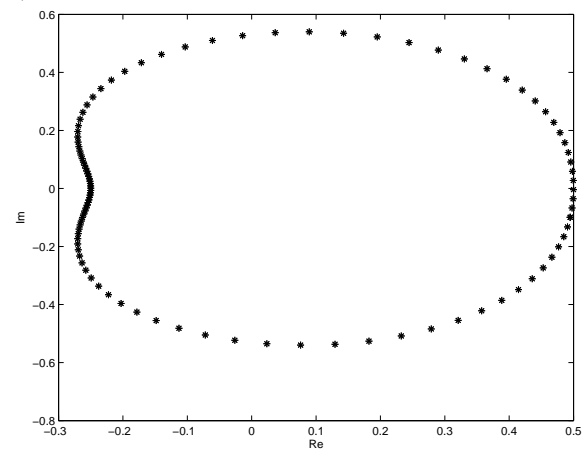

(b)

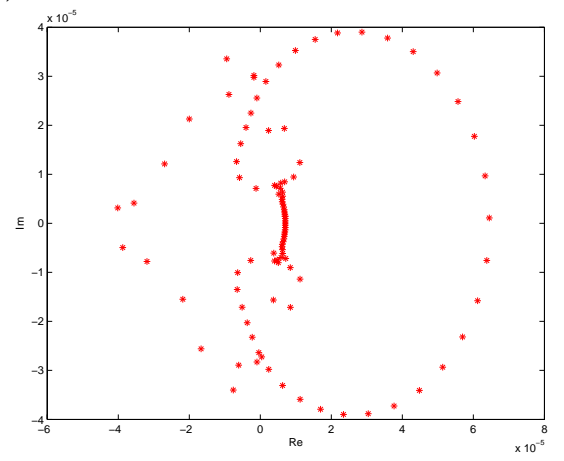

Figure 1: Plot of the solution and the error of the example with $m=1$ and $n=16$. The solution are evaluated at $k=100$ non-collocation points. (a) The solution plot at collocation points. The approximate and the exact solutions are almost identical from the plot. (b) The error plot between the approximate and the exact solution.

method is based on a collocation method and mechanical quadrature rule. The method has been proved to be convergent in Lebesgue spaces. A numerical example illustrated the performance of the new method.

\section{Acknowledgments}

This research was supported by U. S. Civilian Research and Development Foundation (CRDF) and Moldovan Research and Development Association (MRDA) through the Grant LTTG-02. The first author would like to thank the Center for Research in Scientific Computation and the Department of Mathematics, North Carolina State University, for the support and the hospitality during his visit. The second author was supported in part by an ARO grant 43751-MA, and NSF grants DMS-00-73403 and DMS-02-01094.

\section{References}

[1] Beloterkovski S.M., Lifanov I.K., Numerical methods in singular integral equations, Nauka, Moscow, 1985, (in Russian).

[2] Bardzokas D.I., Filshtinsky M.L., Investigation of the direct and inverse piezoeffect in the dynamic problem of electro-elasticity for an unbounded medium with a tunnel opening, ACTA MECHANICA 2002, 155(1), pp. 17-25.

[3] Gabdulalhaev B.G., Direct methods of solving some operational equations . Izvestia vuzov. Mathematics, 1972, 1: 32-43, (in Russian). 
[4] Gabdulhaev B.G., The approximate solution of singular integral equations by the Mechanical quadrature method, Dan USSR 1968, 179(2): pp. 260-263, (in Russian).

[5] Gabdulhaev B. G., Approximation in $H$ spaces and applications, DAN SSSR, 1975, 223(6): pp.1293- 1296, (in Russian).

[6] Gabdulhaev B. G., The finite approximations of singular integrals and direct methods for the solution particular integral and integro-differential equations, Mathematical analysis, VINITI: Moscow, 1980, 18: pp. 251- 307, (in Russian).

[7] Gabdulhaev B.G., Dushkov P.N., The method of mechanical quadratures for the singular integral equations, Izvestia vuzov. Mathematics, 1974, 12: pp. 3-14, (in Russian).

[8] Ivanov V. V., The theory of approximate methods and their application to the numerical solution of singular integral equations, Noordhoff International Publishing, 1976.

[9] Kalandia A.I., Mathematical methods of two- dimensional elasticity, Mir Publishers, 1975 .

[10] Krikunov Iu.V., The general boundary Riemann problem and linear singular integro-differential equation, The scientific notes of the Kazani university, 1956, 116(4):pp. 3-29, (in Russian).

[11] Ladopoulos E.G., Singular integral equations : linear and non-linear theory and its applications in science and engineering, Springer, Berlin, New York, 2000.

[12] Ladopoulos E.G., Finite-part singular integro- differential equation arising in twodimensional aerodynamics, Archives of Mechanics, 1986, 41: pp. 925-936.

[13] Linkov A.M., Boundary Integral Equations in Elasticity Theory, Kluwer Academic: Dordrecht, Boston, 2002.

[14] Lifanov I.K., Singular Integral Equations and Discrete Vortices, Utrecht, the Netherlands, 1996.

[15] Mikhlin S. G.,Morozov N. F., Paukshto M. V., The integral equations of the theory of elasticity, Stuttgart, B.G. Teubner Verlagsgesellschaft, 1995.

[16] Muskhelishvili, N. I., Singular integral equations: boundary problems of function theory and their application to mathematical physics, Leyden, Noordhoff International, 1977.

[17] Muskhelishvili, N. I., Some basic problems of the mathematical theory of elasticity: fundamental equations, plane theory of elasticity, torsion, and bending, Groningen, P. Noordhoff, 1953. 
[18] Prössdorf Siegfried, Some classes of singular equations Amsterdam, New York: North-Holland Pub. Co; New York: sole distributors for the USA and Canada, Elsevier North-Holland, 1978.

[19] Sax R.S., The regional problems for elliptical systems of differential equations, Novosibirsk: University of Novosibirsk, 1975, (in Russian).

[20] Sheremet'ev M.P., Elastic Equilibrium of an Infinite Plate with an Inserted Absolutely Rigid or Elastic Disk, Prikl. Matem. i Mekh., 1952, 16(4): pp. 437-448, (in Russian).

[21] Smirnov V.I.,Lebedev N.A., The constructive theory of the functions of the complex variables, Science: Moscow- Linengrad; 1964, (in Russian).

[22] Zolotarevschi V.A., Seiciuc V.N., The collocation method for the solution of singular integral equations given on the Lyapunov contours, Differential Equations, 1983, 19(6), pp. 1056-1065, (in Russian).

[23] Zolotarevschi V. A., Finite methods of solving the singular integral equations on the closed contours of integration, Stiinta: Kishinev, 1991, (in Russian).

[24] Zolotarevschi V., A., Caraus Iu. N., Direct methods for the solution of singular integro-differential equations on smooth closed arbitrary contours, Rus. Dep. in Mold. NIITAI. nr. 1504- M98, Chisinau, 1998, (in Russian). 